\title{
Post-operative pain management
}

\author{
Lutful Aziz
}

\section{Introduction}

John J. Bonica once said, “Acute pain afflicts millions of patients world-wide. Its effective control remains one of the most important issues in post-operative care today". Modern anesthesia has advanced to a point at which all patients can be guaranteed a pain-free intra-operative period. Unfortunately, we often fall short when it comes to providing post-operative pain relief. It is not surprising; therefore, that what patients often fear most about a surgical procedure is the pain they will experience afterward. Most people suffer from post-operative pain of varying intensity who under go operation. Inadequate treatment of pain causes needless suffering and may develop complications. John J. Bonica again said that, "Inadequate or improper application of knowledge and therapies currently available is certainly one of the most important factors resulting in inadequate relief of pain." The goal for postoperative pain management is to reduce or eliminate pain and discomfort with a minimum of side effects as cheaply as possible. Postoperative pain relief must reflect the needs of each patient and this can be achieved only if many factors are taken into account. These may be summarized as clinical factors, patient-related factors and local factors. ${ }^{1}$ In the final analysis the ultimate determinant of the adequacy of pain relief will be the patient's own perception of pain.

\section{Clinical factors}

The site of the surgery has a profound effect upon the degree of postoperative pain a patient may suffer. Operations on the thorax and upper abdomen are more painful than operations on the lower abdomen which, in turn, are more painful than peripheral operations on the limbs. However, any operation involving a body cavity, large joint surfaces or deep tissues should be regarded as painful. In particular, operations on the thorax or upper abdomen may produce widespread changes in pulmonary function, an increase in abdominal muscle tone and an associated decrease in diaphragmatic function. The result will be an inability to cough and clear secretions which may lead to lung atelectasis and pneumonia aggravated by postoperative bowel distension or tight dressings. ${ }^{2}$

Uncontrolled postoperative pain may activate the sympathetic response of the body which may contribute to morbidity and mortality. Sympathetic activation may increase heart rate, cardiac work and oxygen consumption. ${ }^{3}$ Prolonged pain can reduce physical activity and lead to venous stasis and an increased risk of deep vein thrombosis and consequent pulmonary embolism. ${ }^{1}$ In addition, sympathetic activation delay gut and urinary tract motility which may lead, to postoperative ileus, nausea, vomiting and urinary retention. These problems are unpleasant for the patient and may prolong hospital stay. $^{2}$

Unrelieved pain after surgery can lead to complications, prolonged hospital stay, and delayed recovery. Because of side effects from opioids and differences in response, it is important to use non-pharmacological methods in addition to analgesics to decrease patient discomfort and anxiety. The effects of systematic relaxation reduced their pain and increased their sense of control. ${ }^{4}$ Improvement can be achieved by better education for all staff concerned with the delivery of postoperative pain relief and by making the assessment and recording of pain levels part of the routine management of each patient.

\section{Patient-related factors}

Although it may be possible to predict, to a 
degree, the amount of postoperative pain knowing the site and nature of the surgery, other factors may alter the amount of pain suffered by the individual patient. The nature and intended purpose of the surgery may be important. If the proposed operation will lead to a restoration of normal function, for example, a hernia repair or fixation of a fracture, it is likely to be seen in a positive way by the patient. ${ }^{1}$ Patients who are afraid of anesthesia or surgery may report more pain and this can be very difficult to treat. ${ }^{4}$ Psychogenic pain does not hurt and is very similar to malingering. The benefit of pain relief includes relieving fear, anxiety, and depression and encourages accepting surgical procedures which are necessary have more attention by new generation. It also improves post-operative comfort, mobility, motivation, rate of recovery and nursing workload. ${ }^{1}$

\section{Local Factors}

A major problem in some parts of the world is that certain drugs, such as morphine, which are the mainstay of postoperative pain relief in many places, are not available. In addition, economic factors may mean that techniques of pain relief such as patient-controlled analgesia (PCA) are unavailable and that techniques of regional anesthesia which employ continuous infusions through disposable catheters are impossible. It is no use advocating techniques such as these if they are beyond local resources. It is better to maximize the effective use of local anesthetic techniques and intermittent delivery of such analgesic drugs as are available. ${ }^{1}$

In general, the introduction of new and potential techniques may yield increased benefits in the form of improved recovery and faster discharge from hospital with consequent reductions in the cost of health care. Effective postoperative pain management may be encouraged by education of politicians, administrators, professional colleagues and patients.

\section{Survey}

A 1996 study from Spain (Bosch F. et al) involved a survey of 172 surgical nurses at 4 different centers. Most respondents considered pain to be an unavoidable part of surgery, the dosage of opioid drugs considered as adequate was often too low to be of use in severe pain, excessive fear of inducing drug dependence and most nurses believed that if the patient was pain free at the scheduled time of dosing, the analgesia dose should be missed. A recent UK study compared 78 nurses' and 39 patient' rating of pain following surgery. The pain experienced by patients was still being underestimated, which is consistent with previous research findings. The study also identified some typical misconceptions about pain (Field L, 1996). The patient who uses his/her pain to obtain benefits/preferential treatments does not hurt as much as he/she says and may not hurt at all.

\section{Different Analgesic techniques}

Pain arising from deep or visceral structures requires the use of strong opioids. Appropriate treatment begins with an understanding of the correct drug, route of administration and the mode of action. Early administration will achieve effective analgesic concentrations and make it easier to maintain the therapeutic level of the drug in the blood. ${ }^{1}$ Once a satisfactory level of pain relief has been achieved this can be sustained by regular administration of opioid regardless of whether the intramuscular, subcutaneous, intravenous, oral, sublingual or rectal route is chosen. Administration of adequate doses of analgesic may be inhibited because of side effects, notably nausea and vomiting. In general, effective analgesia can be provided by intramuscular injection despite the recognized drawbacks of this method. Conventional intramuscular delivery of opioid analgesics has the advantage of representing familiar practice and has inherent safety for this reason. The technique 
is inexpensive and the gradual onset of pain relief permits easy assessment of possible overdose. A disadvantage of the method may be that the dose is too large (side effects) or too small (no pain relief). In addition, the injections are painful and the onset of pain relief is delayed while the drug is absorbed. Regional anesthetic techniques used for surgery may have positive respiratory and cardiovascular effects associated with reduced blood loss and excellent pain relief which can improve convalescence. ${ }^{1}$ Clearly, any technique that can be used for the surgical procedure will provide near perfect postoperative pain relief if it can be prolonged beyond the time of the surgery. There are many straightforward local anesthetic techniques which can be continued into the postoperative period to provide effective pain relief. Most of these can be carried out with minimal risk to the patient and include local infiltration of incisions with long-acting local anesthetics, blockade of peripheral nerves or plexuses and continuous block techniques peripherally or centrally. It is a mistake to expect $100 \%$ analgesia in every patient using a local anesthetic technique alone as postoperative pain has many sources. ${ }^{1}$ The true place of local anesthetic techniques is as part of a prepared plan for overall management that employs these techniques in conjunction with appropriate analgesic drugs.

New technologies e.g. Infusion pump, PCA, etc, Value of local and regional anesthesia in blocking nociceptive pain, Concept of "balanced analgesia" and its use in optimized pain management in the post operative period have added new dimensions. ${ }^{5}$

PCA can be administered through intravenous, intramuscular, subcutaneous and also epidural routes. This technique has advantages as it overcomes the wide variation in patients' analgesic requirements allows patients to balance their own comfort with out any side-effect of opioids. PCA provides better patient satisfaction and improved respiratory function than conventional routes for administration of opioids. ${ }^{6}$ The reasons for this are not clear but are probably related to fears of over dosage, the need for contact with members of the hospital staff and the expectation of some pain after surgery. The normal pattern of use is for frequent demands to be made in the initial postoperative period and for these to decrease with time. The total amount of opioid used is less with PCA than with intramuscular delivery. The overall incidence of side effects is about the same with the two techniques but the incidence of respiratory depression is less with PCA.

Modern concept of multimodal analgesia referred as "balance analgesia" is an approach to pain management, should consist of a combination of approaches in order to achieve the best results. ${ }^{5}$ Local anesthetic injections at any site can form part of balanced analgesia where a mixture of techniques provides pain relief. This has the advantage of decreasing the dosage of each drug needed and diminishing the likelihood of side effects. The small delay that results from performance of the blocks is outweighed by the benefit to the patient.

Altered central nervous system sensory processing is a basic mechanism underlying postoperative pain. One such basic mechanism is the alteration of peripheral and central nervous system function. ${ }^{7.8}$ These alterations, which can be excitatory or inhibitory and are termed neuroplasticity, are now considered to play an important role in all areas of acute pain. ${ }^{8-10}$ Prophylactic use of analgesia to reduce their post-operative requirement by reducing noxious input and thereby minimizing hyperexcitablility "wind-up" state of CNS known as pre-emptiva analgesia is another new concept.

Recently, the development in the knowledge of pathophysiology of acute pain and chemical pharmacology of opioids and non-opioids including pharmacokinetics and pharmacodynamics increased the use of these new drugs for post-operative analgesia. 


\section{Pain relief in children ${ }^{1}$}

Management of pain in children is often inadequate and ignored previously with misconception of low intensity of pain. But there is no evidence to support the idea that pain is less intense in neonates and young children due to their developing nervous system. Children tend to receive less analgesia than adults and the drugs are often discontinued sooner. Furthermore, it is simply not true that potent analgesics are dangerous when used in children because of the risks of side effects and addiction. As with all pain, successful management depends upon the identification and treatment of all the factors which contribute, in particular fear and anxiety. In this context, careful explanations to child and parents can be helpful. A major problem in treating pain in children is associated with the difficulty in assessment.

Assessment presents a major challenge, especially in those patients who cannot explain how they feel and who cannot understand the relationship between the treatment and the pain. The worst response is to ignore the presence of pain and the best is to assess the pain and the patient's response to treatment as thoroughly as possible. In very young children observational measures may be helpful, but absence of these signs does not rule out the existence of pain. Assessing simple factors such as whether or not the child is asleep, crying, relaxed, and tense or are responding to their parents may be used to create a cumulative pain score.

\section{Pain relief in the elderly}

The elderly also present special problems in the provision of analgesia. There may be great difficulty in communication and assessment and the choice of analgesic techniques should reflect this. As a general rule the elderly report pain less frequently and require smaller doses of analgesic drugs to achieve adequate pain relief.
Many patients are anxious, however, and this may correlate with increased pain postoperatively. Assessment of pain may be carried out by normal methods and conventional numerical or graphical methods work well. However, impairment of higher intellectual functions may mean that observational techniques similar to those described earlier be needed. When analgesic drugs are given they may not be absorbed as well or metabolized as efficiently. In practical terms, doses of drugs such as NSAIDs and opioids should be reduced because of a decrease in liver metabolism. In addition, since the metabolites of drugs such as morphine and pethidine are excreted by the kidneys, any decrease in renal function may lead to accumulation with repeated doses. The elderly are more likely to be receiving more than one drug for underlying medical conditions and the possibility of drug interaction is greater.

\section{Conclusion}

Acute pain management services (APMS) evolved in response to the desire for improved management of postoperative pain. This will result in improved postoperative analgesia, advancement with new analgesic methods, improved undergraduate and graduate medical education in pain and increased efforts to carry out standardized bench, clinical, and population-based research. ${ }^{11}$ These efforts will provide health care professionals with comprehensive feedback so they may strive for both clinical excellence and professional fulfillment. To apply advanced new analgesic methods found IVPCA, epidural analgesia and peripheral nerve blocks to be the mainstays of therapy on the wards. Baker, ${ }^{12}$ Rawal $^{13}$ and Klein $^{14}$ report a trend towards discharging patients with indwelling nerve block catheters. These initiatives along with improved management of postoperative pain in hospitals may address the high rates of moderate to severe pain being reported by patients after discharge from hospital ${ }^{15,16}$ and may ultimately 
facilitate earlier discharge from hospital and reduce readmission rates in both post surgical and palliative patients.

The future looks bright with all these new techniques and drugs. But again established facts are more safe and beneficial for the patients than anything that is yet to be established. As John Dryden Says, "For the happiness mankind can gain, is not in pleasure but in rest from pain.”

\section{References}

1. Ed Carlton. The management of postoperative pain, practical procedures. Update in Anaesthesia, Issue 7, World Federation of Societies of Anaesthesiologists. 1997;2:1-7.

2. Christofer L, Wu. Acute post-operative pain. Miller's Anaesthesia. Ed Ronald D. Miller $6^{\text {th }}$ edition 2005;2;72: 2729-2762.

3. Warltier DC, Pagel PS, Kersten JR. Approaches to prevention of perioperative myocardial ischemia. Anaesthesiology. 2000;92:253.

4. Roykulcharoen V, Good M. Systematic relaxation to relieve postoperative pain. Journal of Advanced Nursing, October 2004, 48(2), 140-148(9), Blackwell Publishing.

5. David James, Douglas Justins. Acute postoperative pain. A Practice of Anaesthesia ed Wiley and Churchill Davidson's, $7^{\text {th }}$ edition 2005;74:1213-1234.

6. McArdle CS. Continuous and patient controlled analgesic infusions. In: Doyle E (ed) 1998 International Symposium on Pain Control. London: RSM International Congress and Symposium Series No. 123:17-22.
7. Raja SN, Meyer RA, Campbell JN. Peripheral mechanisms of somatic pain. Anesthesiology. 1988;68:571-90.

8. Coderre TJ, Katz J, Vaccarino AL, Melzack R. Contribution of central neuroplasticity to pathological pain: review of clinical and experimental evidence. Pain. 1993;77:362-79.

9. Woolf CJ, Salter MW. Neuronal plasticity: increasing the gain in pain. Science. 2000;288:1765-8.

10. Woolf CJ, Chong MS. Pre-emptive analgesia: treating postoperative pain by preventing the establishment of central sensitization. Anesth Analg. 1993;77:362-79.

11. David H. Goldstein, Elizabeth G. VanDenKerkhof, and William C. Blaine. Acute pain management services have progressed, albeit insufficiently in canadian academic hospitals, regional anesthesia and pain, Can J Anesth. 2004;51:231-235.

12. Baker CJ, Rennie W, Brown R, Kenny S. Home plexus catheters after major limb surgery. Can J Anesth. 2003;50:A20 (abstract).

13. Rawal N, Allvin R, Axelsson K. Patient-controlled regional analgesia (PCRA) at home. Controlled comparison between bupivacaine and ropivacaine brachial plexus analgesia. Anesthesiology. 2002;96:1290-6.

14. Klein SM. Beyond the hospital: continuous peripheral nerve blocks at home (Editorial). Anesthesiology. 2002;96:1283-5.

15. Chung F, Ritchie E, Su J. Postoperative pain in ambulatory surgery 1997;85:986. Anesth Analg. 1997; 85:808-16; Erratum 1997; 85: 986.

16. Collins M, MacDonald V. Managing post-operative pain at home. Canadian Nurse 2000;96:26-9. 